\title{
Generalization of $z$-ideals in right duo rings
}

\author{
Maryam Masoudi-Arani ${ }^{1,2}$ (D), Reza Jahani-Nezhad*1 (D) \\ ${ }^{1}$ Department of Mathematics, University of Kashan, Kashan, Iran \\ ${ }^{2}$ Department of Fundamental Sciences, Faculty of Fadak, Kashan Branch, Technical and Vocational \\ University (TVU), Isfahan, Iran
}

\begin{abstract}
The aim of this paper is to generalize the notion of $z$-ideals to arbitrary noncommutative rings. A left (right) ideal $I$ of a ring $R$ is called a left (right) $z$-ideal if $M_{a} \subseteq I$, for each $a \in I$, where $M_{a}$ is the intersection of all maximal ideals containing $a$. For every two left ideals $I$ and $J$ of a ring $R$, we call $I$ a left $z_{J}$-ideal if $M_{a} \cap J \subseteq I$, for every $a \in I$, whenever $J \nsubseteq I$ and $I$ is a $z_{J}$-ideal, we say that $I$ is a left relative $z$-ideal. We characterize the structure of them in right duo rings. It is proved that a duo ring $R$ is von Neumann regular ring if and only if every ideal of $R$ is a $z$-ideal. Also, every one sided ideal of a semisimple right duo ring is a $z$-ideal. We have shown that if $I$ is a left $z_{J}$-ideal of a $p$-right duo ring, then every minimal prime ideal of $I$ is a left $z_{J}$-ideal. Moreover, if every proper ideal of a $p$-right duo ring $R$ is a left relative $z$-ideal, then every ideal of $R$ is a $z$-ideal.
\end{abstract}

Mathematics Subject Classification (2010). 16D25, 16N20, 16E50, 16D60, 16D99, $16 \mathrm{U} 80$

Keywords. $z$-ideal, duo ring, relative $z$-ideal, semisimple ring, von Neumann regular ring

\section{Introduction}

Throughout this article all rings are associative with identity. The notion of $z$-ideals which are both algebraic and topological objects was first introduced in [6] by Kohls. These ideals play a fundamental role in studying the ideal structure of $\mathrm{C}(\mathrm{X})$, the ring of real-valued continuous functions on a completely regular Hausdorff space X, see [6]. Although in [6], he defined these ideals topologically, in terms of zero-sets, he showed that they can be characterized algebraically. Gillman and Jerison in [4], have proved it to be a powerful tool in the study of both algebraic properties of function rings and topological properties of Tychonoff spaces.

It was Mason [11], who initiated the study of $z$-ideals in arbitrary commutative rings with identity. An ideal $I$ of a commutative ring $R$ is called a $z$-ideal ( $z^{\circ}$-ideal) if for each $a \in I$, the intersection of all maximal ideals (minimal prime ideals) containing $a$

\footnotetext{
*Corresponding Author.

Email addresses: masoudiar@gmail.com (M. Masoudi-Arani), jahanian@kashanu.ac.ir (R. Jahani-Nezhad) Received: 05.03.2019; Accepted: 28.10.2019
} 
is contained in I. A. Rezaei Aliabad and R. Mohamadian in [12], characterized the $z$-ideals and $z^{\circ}$-ideals of formal power series ring on a commutative ring. They showed that if $R$ is a commutative ring, then an ideal $I$ of formal power series ring $R[[x]]$ is a $z$-ideal if and only if $I=(J, x)$, where $J$ is a $z$-ideal of $R$. Also, they characterized a relation between the set of $z^{\circ}$-ideals of $R[[x]]$ and the set of $z^{\circ}$-ideals of $\mathrm{R}$.

Let $I$ and $J$ be two ideals of a commutative ring $R$. $I$ is said to be a $z_{J}$-ideal if $M_{a} \cap J \subseteq I$, for every $a \in I$, where $M_{a}$ is the intersection of all maximal ideals containing $a$. Whenever $J \nsubseteq I$ and $I$ is a $z_{J}$-ideal, we say that $I$ is a relative $z$-ideal. This special kind of $z$-ideals introduced and investigated by F. Azarpanah and A. Taherifar in [2]. They have shown that for any ideal $J$ in $C(X)$, the sum of every two $z_{J}$-ideals is a $z_{J}$-ideal if and only if $X$ is an $F$-space, where the $F$-space is a space for which every finitely generated ideal of $C(X)$ is principal. A space $X$ is called $P$-space if every prime ideal in $\mathrm{C}(\mathrm{X})$ is a $z$-ideal. It is in [2] shown that every principal ideal in $C(X)$ is a relative $z$-ideal if and only if $X$ is a $P$-space. Also, they characterized the space $X$ for which the sum of every two relative $z$-ideals of $C(X)$ is a relative $z$-ideal. If $I$ is an ideal of a semisimple ring and $\operatorname{Ann}(I) \neq 0$, A. R. Aliabad and F. Azarpanah and A. Taherifar in [1], have shown that $I$ is a relative $z$-ideal and the converse is also true for each finitely generated ideal in $C(X)$.

These ideals are also studied further by others in commutative rings. In the following, we present a generalization of $z$-ideals to noncommutative rings and investigate the structure of them in right duo rings, which are rings in which every right ideal is a two-sided ideal. In fact, we generalize the results in [1] to right duo rings. This paper is organized as follows:

In the second section, we study some properties of ideals in right duo rings. In the third section, we shall generalize the concept of $z$-ideal to noncommutative rings and we study their structure in right duo rings. We show that every $z$-ideal of a right duo ring is semiprime. Mason in [10], showed that if $I$ is a $z$-ideal of a semisimple commutative ring, then every minimal prime ideal of $I$ is also a $z$-ideal. In a right duo ring, we consider sufficient conditions that every minimal prime ideal of a $z$-ideal is also a $z$-ideal. We will show that every ideal of von Neumann right duo rings is a $z$-ideal. Also, if every left ideal of a right duo ring $R$ is a $z$-ideal, then $R$ is a von Neumann ring. Furthermore, every left ideal of a semisimple right duo ring is a $z$-ideal.

In the fourth section, we generalize left relative $z$-ideals to noncommutative rings. We define the concept of $p$-right duo rings to obtain equivalent condition to minimal prime ideals of an ideal, and then study left relative $z$-ideals of their rings. We will present sufficient conditions in order that if every proper ideal of a ring $\mathrm{R}$ is a left relative $z$-ideal, then every ideal of $\mathrm{R}$ is a $z$-ideal.

Let us close this section by mentioning some symbols. Let $R$ be a ring and $I$ an ideal of $R$. The set of all prime ideals of $R$ is denoted by $\operatorname{Spec}(R)$. Also, $\operatorname{Min}(I)$ is the set of all minimal prime ideals containing $I$, for each ideal $I$ of $R$, and the Jacobson radical of $R$ is denoted by $\operatorname{rad}(R)$.

\section{Some properties of structure of right duo rings}

Recall that a ring $R$ is called a right duo ring if each right ideal of $R$ is a two sided ideal. We can similarly define the notion of a left duo ring. A ring $R$ is said to be a duo ring if $R$ is a right and left duo ring. Commutative rings and division rings are clearly duo ring. Furthermore, any valuation ring arising from a Krull valuation of a division ring is always duo ring, see [8, Exercise 19.9]. It is easily seen that any finite direct product of a right duo ring is a right duo ring. Proposition 1.1 of [3] says that any homomorphic image of a right duo ring is a right duo ring, and so is any factor ring of it. Gerg Marks in Proposition 5 of [9] shows that any power series ring of a right self injective von Neumann right duo ring is a right duo ring. In particular, the power series ring of a division ring is a right 
duo ring. Further a von Neumann ring which any idempotent element of it is central, is a right duo ring, by [5, Theorem 2.5]. It follows immediately from [3, Proposition 1.1] that for every ideal $I$ of a right (left) duo ring $R, \frac{R}{I}$ is also a right (left) duo ring.

It is well known that in every right duo ring $R, R x R=x R$, for all $x \in R$, and so $R x \subseteq R x R=x R$, see [3]. The following results will be needed in this paper.

Lemma 2.1 ([3]). Let $R$ be a right duo ring and $x \in R$. Then

(1) $R x R=x R$.

(2) $R x \subseteq x R$.

We know that a ring $R$ is called a Dedekind-finite ring if whenever $x, y \in R$ and $x y=1$, then $y x=1$. Now, we assume that $R$ is a right duo ring and $a b=1$, for some $a, b \in R$. Then there exists an element $r \in R$ such that $a b=b r$, by Lemma 2.1. Hence, we have $a=a .1=a(a b)=a(b r)=(a b) r=1 . r=r$, and so $1=b a$. Therefore, every right duo ring is Dedekind-finite, see [8, Theorem 3.2].

It is well known that if $P$ is a prime ideal of a right duo ring and $x y \in P$, then $x \in P$ or $y \in P$, because $x y \in P$ implies that $x R y \subseteq x y R \subseteq P$, by Lemma 2.1. Since $P$ is a prime ideal, we have $x \in P$ or $y \in P$. Therefore, we have the following Lemma:

Lemma 2.2. Let $R$ be a right duo ring and $P$ be a proper ideal of $R$. Then the following statements are equivalent:

(1) $P$ is a prime ideal.

(2) For every $x, y \in R$, if $x y \in P$ then $x \in P$ or $y \in P$.

Therefore, if $P$ is a prime ideal of $R$ and $x^{n} \in P$, for some $x \in R$ and $n \in \mathbb{N}$, then $x \in P$.

Let $R$ be a ring and $I$ be an ideal of $R$. We denote by $\sqrt{I}$ the subset

$$
\left\{r \in R \mid \exists n \in \mathbb{N}, r^{n} \in I\right\}
$$

of $R$. It is easily seen from Lemma 2.1 that if $P$ is a prime ideal of a right duo ring $R$, then $\sqrt{P}=P$.

Lemma 2.3. Let $R$ be a right duo ring and $I$ and $J$ be ideals of $R$. Then

$$
\sqrt{I}+\sqrt{J} \subseteq \sqrt{I+J} .
$$

Proof. Let $a \in \sqrt{I}$ and $b \in \sqrt{J}$. Then there exist $n, m \in \mathbb{N}$ such that $a^{m} \in I$ and $b^{n} \in J$. Now, we claim that $(a+b)^{m+n} \in I+J$. In fact, $(a+b)^{m+n}$ is the sum of $2^{m+n}$ elements of the form $f=c_{1} c_{2} \cdots c_{m+n}$ where each $c_{i}=a$ or $b$. If at least $m$ of these $c_{i}$ s are $a$, then there exists $a^{\prime} \in R$ such that $f=a^{m} a^{\prime}$, by Lemma 2.1, and so $f \in I$, because $a^{m} \in I$. If the number of the $c_{i}=a$ is smaller than $m$, then at least $n$ of them are $b$, and hence there exists $b^{\prime} \in R$ such that $f=b^{n} b^{\prime}$, by Lemma 2.1. Thus $f \in J$, because $b^{n} \in J$. Therefore $(a+b)^{n+m} \in I+J$.

Proposition 2.4. Let $R$ be a right duo ring and $I$ be an ideal of $R$. Then $\sqrt{I}$ is an ideal of $R$.

Proof. Clearly, $0 \in \sqrt{I}$. If $a, b \in \sqrt{I}$, then $a+b \in \sqrt{I}$, by Lemma 2.3. Now, assume that $a \in \sqrt{I}$ and $r \in R$. Hence there exists $n \in \mathbb{N}$ such that $a^{n} \in I$, and so there exists an element $r^{\prime} \in R$ such that $(r a)^{n}=a^{n} r^{\prime} \in I$, by Lemma 2.1. Therefore $r a \in \sqrt{I}$ and similarly we show that $a r \in \sqrt{I}$.

Let $R$ be a ring and $a \in R$. The intersection of all maximal ideals of $R$ containing $a$ will be denoted by $M_{a}$. We set $M_{a}=R$ when $a$ is a unit.

Lemma 2.5. Let $R$ be a right duo ring and $a, b \in R$. Then $M_{a b}=M_{a} \cap M_{b}$. In particular, if $a \in R$ we conclude that $M_{a^{n}}=M_{a}$, for every $n \in \mathbb{N}$. 
Proof. Clearly, for every $x \in M_{a}$ we have $M_{x} \subseteq M_{a}$. Thus $M_{a b} \subseteq M_{a}$ and $M_{a b} \subseteq M_{b}$, and so $M_{a b} \subseteq M_{a} \cap M_{b}$, for each $a, b \in R$. Conversely, let $x \in M_{a} \cap M_{b}$. We show that every maximal ideal containing $a b$ is also containing $x$. Assume that $N$ is a maximal ideal of $R$ such that $a b \in N$. Then $a \in N$ or $b \in N$, by Lemma 2.2. If $a \in N$, then $x \in M_{a} \cap M_{b} \subseteq M_{a} \subseteq N$. If $b \in N$, then $x \in M_{b} \subseteq N$. Therefore $x \in N$. Thus $M_{a} \cap M_{b} \subseteq M_{a b}$, and consequently $M_{a b}=M_{a} \cap M_{b}$.

\section{Generalization of $z$-ideals in a right duo ring}

The $z$-ideals are studied further in commutative rings. These ideals are useful concept in studying the ideal structure of the ring $C(X)$ of continuous real-valued functions on a topological space $X$. In the following, we shall present a generalization of $z$-ideals to noncommutative rings.

Definition 3.1. A left (right) ideal $I$ of a ring $R$ is called a left (right) $z$-ideal if $M_{a} \subseteq I$, for all $a \in I$.

In the following we show that every one sided $z$-ideal is an ideal.

Proposition 3.2. Let $R$ be a ring and $I$ be a left (right) $z$-ideal of $R$. Then $I$ is an ideal of $R$.

Proof. Let $I$ be a left $z$-ideal of $R$ and $a \in I$. If $N$ is a maximal ideal of $R$ containing $a$, then $a r \in N$ for every $r \in R$. Thus $M_{a r} \subseteq M_{a}$. On the other hand, since $I$ is a left $z$-ideal, we have $M_{a} \subseteq I$. Therefore, $a r \in M_{a r} \subseteq M_{a} \subseteq I$, and so $a r \in I$. Hence $I$ is an ideal.

Here in after a left (right) $z$-ideal of a ring is called a $z$-ideal, by Proposition 3.2.

Example 3.3. Every intersection of maximal ideals of a $\operatorname{ring} R$ is a $z$-ideal. In fact, every intersection of $z$-ideals is a $z$-ideal.

Lemma 3.4. Let $R$ be a ring and $I$ be a left (right) ideal of $R$. Then the following statements are equivalent:

(1) I is a z-ideal.

(2) For every $a \in R$ and $b \in I$, if $M_{a} \subseteq M_{b}$ then $a \in I$.

Proof. $1 \Rightarrow 2$. Let $a \in R$ and $b \in I$. Since $I$ is a $z$-ideal and $b \in I$, we have $M_{b} \subseteq I$. Hence, if $M_{a} \subseteq M_{b}$, then $a \in M_{a} \subseteq M_{b} \subseteq I$, and so $a \in I$.

$2 \Rightarrow 1$. Let $I$ be a left ideal and $a \in I$. For each $x \in M_{a}$, we have $M_{x} \subseteq M_{a}$. By hypothesis $x \in I$, and so $M_{a} \subseteq I$. Therefore $I$ is a $z$-ideal.

Let $R$ be a ring and $I$ be a left (right) ideal of $R$. The intersection of all $z$-ideals containing $I$ will be denoted by $I_{z}$. For each element $a \in I_{z}$ and for every $z$-ideal $J$ of $R$ containing $I$, we have $a \in J$. Then $M_{a} \subseteq J$, and so $M_{a} \subseteq I_{z}$. Therefore, we have the following Lemma:

Lemma 3.5. For every left (right) ideal I of a ring $R$, the intersection of all z-ideals containing $I$, which is denoted by $I_{z}$, is a z-ideal. In particular, $I_{z}$ is the smallest $z$-ideal containing $I$.

Lemma 3.6. Let $R$ be a ring. Then the following statements hold.

(1) For every left ideals $I$ and $J$ of $R$, if $I \subseteq J$, then $I_{z} \subseteq J_{z}$.

(2) If $\left\{I_{\lambda}\right\}_{\lambda \in \Lambda}$ is any family of left ideals of $R$, then

$$
\left(\bigcap_{\lambda \in \Lambda} I_{\lambda}\right)_{z} \subseteq \bigcap_{\lambda \in \Lambda}\left(I_{\lambda}\right)_{z} .
$$

Proof. 1. Since every $z$-ideal containing $J$ contains $I$, we see $I_{z} \subseteq J_{z}$.

2. For every $\mu \in \Lambda$, we have $\bigcap_{\lambda \in \Lambda} I_{\lambda} \subseteq I_{\mu}$. Hence our claim is true, by part (1). 
It is immediate that for every $z$-ideal $I$, we have $I_{z}=I$. In the next two Propositions, we study the structure of $z$-ideals in right duo rings.

Proposition 3.7. Let $R$ be a right duo ring and $I$ be an ideal of $R$. Then $I \subseteq \sqrt{I} \subseteq I_{z}$.

Proof. Clearly, $I \subseteq \sqrt{I}$. Now, we assume that $x \in \sqrt{I}$ and $J$ is a $z$-ideal containing $I$. Thus there is a positive integer $n$ such that $x^{n} \in I \subseteq J$. Hence $x \in M_{x}=M_{x^{n}} \subseteq J$, by Lemma 2.5. Therefore $x \in I_{z}$, and so $\sqrt{I} \subseteq I_{z}$.

Recall that a proper ideal $I$ of a ring $R$ is said to be a semiprime ideal if for every ideal $J$ of $R, J^{2} \subseteq I$ implies that $J \subseteq I$. As an immediate consequence of Proposition 3.7 and [7, Theorem 10.11], we get the following result

Corollary 3.8. Let $R$ be a right duo ring and $I$ be a z-ideal of $R$. Then $\sqrt{I}=I$. In particular, $I$ is a semiprime ideal of $R$.

Proposition 3.9. Let $R$ be a right duo ring and $I$ be an ideal of $R$. Then the following statements hold.

(1) $(\sqrt{I})_{z}=I_{z}$.

(2) If $I$ is a $z$-ideal, then $(\sqrt{I})_{z}=I$.

(3) $\sqrt{I_{z}}=(\sqrt{I})_{z}$.

Proof. 1. Every z-ideal containing $\sqrt{I}$ also contains $I$. Therefore $I_{z} \subseteq(\sqrt{I})_{z}$. Conversely, Proposition 3.7 gives $\sqrt{I} \subseteq I_{z}$. This means that $I_{z}$ is a $z$-ideal containing $\sqrt{I}$. Thus $(\sqrt{I})_{z} \subseteq I_{z}$, and consequently $(\sqrt{I})_{z}=I_{z}$.

2. Since $I$ is a $z$-ideal, we have $I_{z}=I$. The proof is completed by (1).

3. We know that $I_{z}$ is a $z$-ideal of $R$. Corollary 3.8 yields $\sqrt{I_{z}}=I_{z}$. Therefore $\sqrt{I_{z}}=(\sqrt{I})_{z}$, by $(1)$.

The following Proposition is a generalization of [11, Proposition 3.1] to noncommutative case.

Proposition 3.10. Let $R$ be a right duo ring. Then the following statements are equivalent:

(1) For any $z$-ideals $I$ and $J, I+J$ is a $z$-ideal.

(2) For any ideals $I$ and $J,(I+J)_{z}=I_{z}+J_{z}$.

(3) The sum of any nonempty family of $z$-ideals is a $z$-ideal.

(4) For every nonempty family $\left\{I_{\alpha}\right\}_{\alpha \in A}$ of ideals,

$$
\left(\sum_{\alpha \in A} I_{\alpha}\right)_{z}=\sum_{\alpha \in A}\left(I_{\alpha}\right)_{z}
$$

Proof. $1 \Rightarrow 2$. Since $I_{z}$ and $J_{z}$ are $z$-ideals, $I_{z}+J_{z}$ is a $z$-ideal containing $I+J$, by hypothesis. Hence $(I+J)_{z} \subseteq I_{z}+J_{z}$. It follows from Lemma 3.6 that $I_{z}+J_{z} \subseteq(I+J)_{z}$. Therefore $(I+J)_{z}=I_{z}+J_{z}$.

$2 \Rightarrow 3$. Let $\left\{I_{\alpha}\right\}_{\alpha \in A}$ be a family of $z$-ideals and $a \in \sum_{\alpha \in A} I_{\alpha}$. Then there exists a finite subset $F$ of $A$ such that $a \in \sum_{\alpha \in F} I_{\alpha}$. Since $I_{\alpha}$ is a $z$-ideal, we have $\left(I_{\alpha}\right)_{z}=I_{\alpha}$, for every $\alpha \in F$. A simple induction argument shows that

$$
\left(\sum_{\alpha \in F} I_{\alpha}\right)_{z}=\sum_{\alpha \in F}\left(I_{\alpha}\right)_{z}=\sum_{\alpha \in F} I_{\alpha} .
$$

Consequently, $\sum_{\alpha \in F} I_{\alpha}$ is a $z$-ideal, and so

$$
M_{a} \subseteq \sum_{\alpha \in F} I_{\alpha} \subseteq \sum_{\alpha \in A} I_{\alpha} .
$$


Therefore $\sum_{\alpha \in A} I_{\alpha}$ is a $z$-ideal.

$3 \Rightarrow 4$. Let $\left\{I_{\alpha}\right\}_{\alpha \in A}$ be a family of ideals. Since $I_{\beta} \subseteq \sum_{\alpha \in A} I_{\alpha}$, for all $\beta \in A$, we have $\left(I_{\beta}\right)_{z} \subseteq\left(\sum_{\alpha \in A} I_{\alpha}\right)_{z}$, for all $\beta \in A$, by Lemma 3.6. Therefore

$$
\sum_{\alpha \in A}\left(I_{\alpha}\right)_{z} \subseteq\left(\sum_{\alpha \in A} I_{\alpha}\right)_{z} .
$$

Since $\left(I_{\alpha}\right)_{z}$ is a $z$-ideal containing $I_{\alpha}$, for all $\alpha \in A$, we may conclude from assumption that $\sum_{\alpha \in A}\left(I_{\alpha}\right)_{z}$ is a $z$-ideal containing $\sum_{\alpha \in A} I_{\alpha}$. Hence

$$
\left(\sum_{\alpha \in A} I_{\alpha}\right)_{z} \subseteq \sum_{\alpha \in A}\left(I_{\alpha}\right)_{z} .
$$

Therefore

$$
\left(\sum_{\alpha \in A} I_{\alpha}\right)_{z}=\sum_{\alpha \in A}\left(I_{\alpha}\right)_{z} .
$$

$4 \Rightarrow 1$. If $I$ and $J$ are $z$-ideals, then $I_{z}=I$ and $J_{z}=J$. By hypothesis, we have $(I+J)_{z}=I_{z}+J_{z}$. Therefore $(I+J)_{z}=I_{z}+J_{z}=I+J$, and so $I+J$ is a $z$-ideal.

Lemma 3.11. Let $R$ be a right duo ring and $P$ be a prime ideal of $R$. Let $n \in \mathbb{N}$, $I_{1}, \ldots, I_{n-1}$ be ideals and $I_{n}$ be a left ideal of $R$. Then the following statements are equivalent:

(1) $I_{j} \subseteq P$, for some $1 \leq j \leq n$.

(2) $\bigcap_{i=1}^{n} I_{i} \subseteq P$.

(3) ${ }_{1} I_{1} I_{2} \cdots I_{n} \subseteq P$.

Proof. $1 \Rightarrow 2 . \bigcap_{i=1}^{n} I_{i} \subseteq I_{j} \subseteq P$.

$2 \Rightarrow 3$. Since $I_{n}$ is a left ideal of $R$, we have $I_{1} I_{2} \cdots I_{n} \subseteq I_{n}$. On the other hand, $I_{i}$ is an ideal, for every $1 \leq i \leq n-1$, and hence $I_{1} I_{2} \cdots I_{n} \subseteq I_{i}$, for all $1 \leq i \leq n$. Thus $I_{1} I_{2} \cdots I_{n} \subseteq \bigcap_{i=1}^{n} I_{i} \subseteq P$.

$3 \Rightarrow 1$. Suppose that $I_{i} \nsubseteq P$ and $x_{i} \in I_{i} \backslash P$, for every $1 \leq i \leq n$. Thus

$$
x_{1} x_{2} \cdots x_{n} \in I_{1} I_{2} \cdots I_{n} \subseteq P
$$

which yields $x_{j} \in P$, for some $1 \leq j \leq n$, by Lemma 2.2. This contradicts the choice of $x_{j}$.

Proposition 3.12. Let $R$ be a right duo ring and $I$ an ideal of $R$. If $I$ is a finite intersection of maximal ideals of $R$, then any minimal prime ideal of $I$ is a $z$-ideal.

Proof. Since $I$ is a finite intersection of maximal ideals, Lemma 3.11 implies that any minimal prime ideal of $I$ is a maximal ideal. Hence each minimal prime ideal of $I$ is a $z$-ideal.

Recall from [7, Definition 10.3] that a nonempty set $S$ of a ring $R$ is said to be $m$-system if for any $a, b \in S$, there exists $r \in R$ such that $a r b \in S$.

Theorem 3.13. Let $R$ be a right duo ring, $I$ a $z$-ideal of $R$ and $Q$ be a minimal prime ideal of $I$. If for every $a, b \in R$ with $a \notin Q$, there exists $r \in R \backslash Q$ such that $a b=b r$, then $Q$ is a $z$-ideal.

Proof. Suppose $Q$ is not $z$-ideal. Then there exist elements $a \in R \backslash Q$ and $b \in Q$ such that $M_{a} \subseteq M_{b}$, by Lemma 3.4. We now assume that

$$
S=(R \backslash Q) \cup\left\{b^{n} c \mid n \in \mathbb{N}, c \in R \backslash Q\right\} .
$$


We first prove that $r_{1} r_{2} \in S$, for every $r_{1}, r_{2} \in S$. Consider $r_{1}, r_{2} \in S$.

- If $r_{1}, r_{2} \in R \backslash Q$, then $r_{1} r_{2} \notin Q$, by Lemma 2.2 , and so $r_{1} r_{2} \in S$.

- If there are $c_{1}, c_{2} \in R \backslash Q$ such that $r_{1}=b^{n} c_{1}$ and $r_{2}=b^{m} c_{2}$, for some positive integers $n$ and $m$, then $c_{1} b^{m}=b^{m} r$, for some $r \in R \backslash Q$, by hypothesis. Therefore

$$
r_{1} r_{2}=b^{n} c_{1} b^{m} c_{2}=b^{(n+m)} r c_{2} \in S
$$

because $r, c_{2} \in R \backslash Q$, and so $r c_{2} \notin Q$, by Lemma 2.2 .

- If $r_{1} \in R \backslash Q$ and $r_{2}=b^{n} c$, for some $n \in \mathbb{N}$ and $c \in R \backslash Q$, then there exists an element $r \in R \backslash Q$ such that $r_{1} b^{n}=b^{n} r$, by hypothesis. This yields

$$
r_{1} r_{2}=r_{1} b^{n} c=b^{n} r c .
$$

Since $r c \notin Q$, we have $r_{1} r_{2} \in S$. Also, we see that $r_{2} r_{1}=b^{n} c r_{1} \in S$. Hence for every $r_{1}, r_{2} \in S$ we have $r_{1} r_{2} \in S$. Therefore $S$ is an $m$-system of $R$.

Now, we show that $I \cap S=\emptyset$. If $x \in I \cap S$, then $x \in I \subseteq Q$ and $x \in S$. Hence $x=b^{n} c$, for some $n \in \mathbb{N}$ and $c \in R \backslash Q$. From Lemma 2.5 we see that

$$
a c \in M_{a c}=M_{a} \cap M_{c} \subseteq M_{b} \cap M_{c}=M_{b^{n}} \cap M_{c}=M_{x} \subseteq I
$$

because $M_{a} \subseteq M_{b}$ and $I$ is a $z$-ideal. This yields $a c \in Q$. Hence $a \in Q$ or $c \in Q$, by Lemma 2.2. This contradicts the choice of $a$ and $c$. Therefore $I \cap S=\emptyset$. By Zorn's Lemma, there exists an ideal $I \subseteq P$ which is maximal with respect to being disjoint from $S$. From [7, Proposition 10.5] it follows that $P$ is a prime ideal. Since $S \cap P=\emptyset$ and $b \in S$, we have $I \subseteq P \varsubsetneqq Q$. However, this contradicts our assumption that $Q$ is a minimal prime ideal of $I$. Therefore $Q$ is a $z$-ideal.

We know that the Jacobson radical of a ring $R$, which denoted by $\operatorname{rad}(R)$, is the intersection of all maximal right (or left) ideals of $R$. Now, if $R$ is a right (or left) duo ring, then every maximal right (or left) ideal is a maximal ideal. Therefore, if $R$ is a right (or left) duo ring, then we can say that $\operatorname{rad}(R)$ is the intersection of all maximal ideals of $R$.

Example 3.14. Let $D$ be a division ring and $\mathbb{C}$ be the field of complex numbers. Let $R=D \times \mathbb{C}[x]$. We know that $R$ is a duo ring. If $f \in \operatorname{rad}(\mathbb{C}[x])$, then $1-x f$ is a unit of $\mathbb{C}[x]$, by $[7$, Lemma 4.1], which yields $f=0$. Hence $\operatorname{rad}(\mathbb{C}[x])=0$. This implies that $\operatorname{rad}(R)=\operatorname{rad}(D) \times \operatorname{rad}(\mathbb{C}[x])=0$, and so $I=\{0\}$ is a $z$-ideal of $R$. If $P$ is a prime ideal of $R$, then $P=0 \times \mathbb{C}[x]$ or $P=D \times Q$, where $Q$ is a prime ideal of $\mathbb{C}[x]$. obviously, $D \times 0$ and $0 \times \mathbb{C}$ are minimal prime ideals of $I$ which $0 \times \mathbb{C}$ is maximal, and so is a $z$-ideal. Consider $(a, f),(c, g) \in R$ such that $(a, f) \notin D \times 0$. It is clear that $a c=c r$, for some $r \in D$. Thus

$$
(a, f)(c, g)=(a c, f g)=(c r, g f)=(c, g)(r, f) .
$$

Since $(a, f) \notin D \times 0$, we have $f \neq 0$, and so $(r, f) \notin D \times 0$. Therefore $D \times 0$ is a $z$-ideal, by Theorem 3.13 .

Proposition 3.15. Let $R$ be a right duo ring and $I$ be a left ideal of $R$. Then $\left(I^{n}\right)_{z}=I_{z}$, for every $n \in \mathbb{N}$.

Proof. Clearly, $\left(I^{n}\right)_{z} \subseteq I_{z}$. For every $x \in I$, we have $x^{n} \in I^{n} \subseteq\left(I^{n}\right)_{z}$, and so $M_{x^{n}} \subseteq\left(I^{n}\right)_{z}$. From Lemma 2.5, we see that $x \in M_{x}=M_{x^{n}} \subseteq\left(I^{n}\right)_{z}$. Hence $I \subseteq\left(I^{n}\right)_{z}$. By Lemma 3.5, $\left(I^{n}\right)_{z}$ is a $z$-ideal, and so $I_{z} \subseteq\left(I^{n}\right)_{z}$. Therefore $\left(I^{n}\right)_{z}=I_{z}$.

Recall that a ring $R$ is said to be a von Neumann regular ring if for any $a \in R$, there exists an element $r \in R$ such that $a=$ ara. Furthermore, for any ideal $I$ of a von Neumann regular ring $R$, it is clear that $\frac{R}{I}$ is also a von Neumann regular ring. Therefore, we have

Proposition 3.16. Let $R$ be a right (or left) duo ring. If $R$ is a von Neumann regular ring, then every ideal of $R$ is a $z$-ideal. 
Proof. Let $I$ be a proper ideal of $R$. Since $\frac{R}{I}$ is a von Neumann regular ring, we have $\operatorname{rad}\left(\frac{R}{I}\right)=0$, by [7, Corollary 4.24]. On the other hand, $\frac{R}{I}$ is also a right (or left) duo ring. Thus $\operatorname{rad}\left(\frac{R}{I}\right)$ is the intersection of all maximal ideals of $\frac{R}{I}$. Hence $I$ is the intersection of all maximal ideal of $R$ containing $I$, and so $I$ is a $z$-ideal.

Proposition 3.17. Let $R$ be a right duo ring. If every left ideal of $R$ is a $z$-ideal, then $R$ is a von Neumann regular ring.

Proof. Let $a \in R$ and $I=R a$. By hypothesis, $I$ is a $z$-ideal, and so $I_{z}=I$. Hence $\left(I^{2}\right)_{z}=I_{z}=I$, by Proposition 3.15. On the other hand, from Lemma 2.1, we see that $I^{2}=R a R a=a R a$. Since $I^{2}$ is a left ideal, $I^{2}$ is a $z$-ideal, and so $\left(I^{2}\right)_{z}=I^{2}$. Hence $I^{2}=\left(I^{2}\right)_{z}=I_{z}=I$. Then we may conclude from $a \in I=I^{2}=a R a$ that there exists an element $r \in R$ such that $a=$ ara. Therefore $R$ is a von Neumann regular ring.

The following result, which is a generalization of [10, Theorem 1.2] to noncommutative case, follows immediately from Proposition 3.16 and Proposition 3.17.

Corollary 3.18. Let $R$ be a duo ring. Then $R$ is a von Neumann regular ring if and only if every ideal of $R$ is a $z$-ideal.

Recall that if $I$ and $J$ are two left ideals of a ring $R$, then the subset $\{x \in R \mid x I \subseteq J\}$ is denoted by $\left(J:_{l} I\right)$. It is easily seen that $\left(J:_{l} I\right)$ is an ideal of $R$. In particular, for each left ideal $I$, the subset $\left(0:_{l} I\right)$, which will be denote by $A n n_{l}(I)$, is also an ideal of $R$. We call it the left annihilator of $I$.

Proposition 3.19. Let $I$ and $J$ be two left ideals of a right duo ring $R$. If $J$ is a $z$-ideal, then $\left(J:_{l} I\right)$ is a $z$-ideal of $R$.

Proof. By Lemma 3.4, it is sufficient to show that for every $a \in R$ and $b \in\left(J:_{l} I\right)$, if $M_{a} \subseteq M_{b}$ then $a \in\left(J:_{l} I\right)$. Now, we assume that $a \in R, b \in\left(J:_{l} I\right)$ and $M_{a} \subseteq M_{b}$. Thus for every $x \in I$, we have $b x \in J$. Moreover

$$
M_{a x}=M_{a} \cap M_{x} \subseteq M_{b} \cap M_{x}=M_{b x}
$$

by Lemma 2.5. Since $b x \in J$ and $J$ is a $z$-ideal, $M_{b x} \subseteq J$, and so $a x \in M_{a x} \subseteq M_{b x} \subseteq J$. Therefore $a \in\left(J:_{l} I\right)$.

Lemma 3.20. If $e$ is an idempotent element of a right duo ring $R$, then

$$
R e=A n n_{l}(R(1-e)) .
$$

Proof. For every $r \in R$, we have $r e R(1-e) \subseteq r e(1-e) R=0$, by Lemma 2.1. Hence $R e \subseteq A n n_{l}(R(1-e))$. We now assume that $r \in A n n_{l}(R(1-e))$. Thus $r-r e=r(1-e)=0$, and so $r=r e \in R e$.

From [7, Theorem 2.5], it follows that every right ideal of a ring $R$ is a direct summand of $\mathrm{R}$ if and only if every left ideal of $R$ is a direct summand of $\mathrm{R}$. A ring satisfying these equivalent conditions is called a semisimple ring.

Proposition 3.21. Let $R$ be a semisimple right duo ring. Then every one sided ideal of $R$ is an ideal.

Proof. Since $R$ is a right duo ring, every right ideal of $R$ is an ideal. By [7, Theorem $4.25]$, semisimple rings are exactly the left Noetherian von Neumann regular rings. Let $I$ be a left ideal of $R$. Since $R$ is left Noetherian, every left ideal of $R$ is finitely generated, and so $I=R e$, for an idempotent element $e$ of $R$, by using the characterization (3) of [7, Theorem 4.23]. Hence $I=A n n_{l}(R(1-e))$, by Lemma 3.20, and consequently $I$ is an ideal of $R$. 
As observed in the proof of Proposition 3.21, every ideal of a semisimple right duo ring is an annihilator of a left ideal. On the other hand, we know from [7, Theorem 4.25] that $\operatorname{rad}(R)=0$, for every semisimple $\operatorname{ring} R$, and hence the zero ideal of $R$ is a $z$-ideal. Now, we may by using Proposition 3.19 conclude that the following result.

Corollary 3.22. Every ideal of a semisimple right duo ring is a z-ideal.

\section{Relative $z$-ideals in a right duo ring}

The main goal of this section is to introduce left relative $z$-ideals. We define the concept of $p$-right duo rings to obtain equivalent condition to minimal prime ideals of an ideal, and then study left relative $z$-ideals of their rings. Finally, we prove that if every proper ideal of a $p$-right duo ring $\mathrm{R}$ is a left relative $z$-ideal, then every ideal of $\mathrm{R}$ is a $z$-ideal.

Definition 4.1. Let $J$ be a left ideal of a ring $R$. A left ideal $I$ of $R$ is said to be a left $z_{J}$-ideal if $M_{a} \cap J \subseteq I$, for every $a \in I$. Whenever, for a left ideal $I$, there exists a left ideal $J$ such that $J \nsubseteq I$ and $I$ is a left $z_{J}$-ideal, we say that $I$ is a left relative $z$-ideal and $J$ is called a $z$-factor of $I$.

Recall that a ring $R$ is said to be a reduced ring if $R$ has no nonzero nilpotent element. In the following, we introduce a class of left relative $z$-ideals in a right duo ring. Before giving it, let us state the following Lemma which follows immediately from Lemma 2.2.

Lemma 4.2. For each right duo ring $R$, if $\operatorname{rad}(R)=0$, then $R$ is a reduced ring.

Proposition 4.3. Let $R$ be a right duo ring with $\operatorname{rad}(R)=0$. If $I$ is a left ideal of $R$ such that $A n n_{l}(I) \neq 0$, then $I$ is a left relative $z$-ideal.

Proof. First, we show that $M_{a} \cap A n n_{l}(I)=0$, for every $a \in I$. Suppose that $x \in M_{a} \cap \operatorname{Ann}_{l}(I)$. Then $M_{x} \subseteq M_{a}$ and $x a \in x I=0$, for every $a \in I$. From Lemma 2.5, it thus follows that

$$
x \in M_{x}=M_{x} \cap M_{a}=M_{x a}=M_{0}=\operatorname{rad}(R)=0 .
$$

Hence $M_{a} \cap A n n_{l}(I)=0$. We now put $J=A n n_{l}(I)$, and show that $J \nsubseteq I$. If $J \subseteq I$, then $J^{2} \subseteq J I=A n n_{l}(I) I=0$. Thus $J^{2}=0$, and so $J=0$, because $R$ is a reduced ring, by Lemma 4.2. But this contradicts the assumption that $J=A n n_{l}(I) \neq 0$. Therefore $J \nsubseteq I$, and so $I$ is a left relative $z$-ideal.

Definition 4.4. A right duo ring $R$ is called a $p$-right duo ring if for every prime ideal $P$ of $R$ and every elements $a, b \in R$, which $a \notin P$, there exists $r \in R \backslash P$ such that $a b=b r$.

In the following, we give some examples of $p$-right duo rings.

Proposition 4.5. Let $R$ be a prime right duo ring. If $R$ has a unique nonzero prime ideal, then $R$ is a p-right duo ring.

Proof. Let $P$ be the unique nonzero prime ideal of $R$ and $a, b \in R$ such that $a \notin P$. If $b=0$, then $a b=b 1$. Now, we assume that $b \neq 0$. Since $R$ is a right duo ring, $a b=b r$, for some $r \in R$. On the other hand, $P$ is the unique nonzero prime ideal of $R$ and $a \notin P$. Thus $a$ is a unit element of $R$ which yields $b=a^{-1} b r=b r^{\prime} r$, for some $r^{\prime} \in R$. It follows $b\left(1-r^{\prime} r\right)=0$. Since $R$ is a prime right duo ring and $b \neq 0$, we have $r^{\prime} r=1$. Therefore $r \notin P$.

Example 4.6. Let $D$ be a division ring and $R=D \times \mathbb{Z}$. We show that $R$ is a $p$-right duo ring. It is easily seen that $R$ is a right duo ring. If $P$ is a prime ideal of $R$, then $P=D \times 0$, or $P=0 \times \mathbb{Z}$ or $P=D \times p \mathbb{Z}$, for some prime number $p$. We assume that $(a, b),(c, d) \in R$ and $(a, b) \notin P$. It is clear that $a c=c r$, for some $r \in D$. Thus

$$
(a, b)(c, d)=(a c, b d)=(c r, d b)=(c, d)(r, b) .
$$


We consider the following three cases:

1. If $P=D \times 0$, then $b \neq 0$, because $(a, b) \notin P$, and so $(r, b) \notin P$.

2. If $P=0 \times \mathbb{Z}$, then $a \neq 0$, because $(a, b) \notin P$. Now, if $c \neq 0$, then $r \neq 0$, and so $(r, b) \notin P$, and if $c=0$, we have

$$
(a, b)(c, d)=(0, b d)=(0, d b)=(c, d)(1, b)
$$

which $(1, b) \notin P$.

3. If $P=D \times p \mathbb{Z}$, for some prime number $p$, then $p \nmid b$, because $(a, b) \notin P$, and so $(r, b) \notin P$.

Proposition 4.7. Let $R$ be a p-right duo ring with $\operatorname{rad}(R)=0$ and $P$ be a prime ideal of $R$. Let $\Gamma$ be the set of all $z$-ideals of $R$ contained in $P$. Then $\Gamma$ (partially ordered by inclusion) has a maximal element. Furthermore, every maximal element of $\Gamma$ is a prime $z$-ideal of $R$.

$\boldsymbol{P r o o f}$. Since $\operatorname{rad}(R)=0$, the zero ideal of $R$ is a $z$-ideal, and so $\Gamma \neq \emptyset$. If $P$ is a $z$-ideal, then clearly $P$ is the only maximal element of $\Gamma$. We now assume that $P$ is not $z$-ideal. If $\Sigma$ is a chain in $\Gamma$, then it is quite obvious that $\bigcup_{I_{\alpha} \in \Sigma} I_{\alpha}$ is a $z$-ideal contained in $P$. Therefore $\Gamma$ has a maximal element $J$, by Zorn's Lemma. Hence $J \subsetneq P$, because $P$ is not $z$-ideal. Suppose that $Q$ is a minimal prime ideal of $J$ such that $J \subseteq Q \subseteq P$. Theorem 3.13 implies that $\mathrm{Q}$ is a $z$-ideal, because $R$ is a $p$-right duo ring, and so $Q \in \Gamma$. Since $J$ is a maximal element in $\Gamma$, we have $J=Q$. Therefore $J$ is a prime $z$-ideal.

Proposition 4.8. Let $R$ be a ring and $I$ be a proper ideal of $R$. Let

$$
\Gamma=\{S \subseteq R \mid S \text { is an } m \text { - system and } S \cap I=\emptyset\} .
$$

If $P$ is a prime ideal of $R$, then $P \in \operatorname{Min}(I)$ if and only if $R \backslash P$ is a maximal element of $\Gamma$.

Proof. We know from [7, Corollary 10.4] that an ideal $P$ of $R$ is prime if and only if $R \backslash P$ is an $m$-system. Therefore, if $T=R \backslash P$ is a maximal element of $\Gamma$, then $P$ is a prime ideal of $R$. Also, $T \cap I=\emptyset$ implies that $I \subseteq P$. Now, we assume that there exists a prime ideal $Q$ of $R$ such that $I \subseteq Q \subseteq P$. It follows that $(R \backslash Q) \cap I=\emptyset$ and $R \backslash Q$ is an m-system, by [7, Corollary 10.4]. Thus $R \backslash Q \in \Gamma$. Since $T \subseteq R \backslash Q$ and $T$ is a maximal element of $\Gamma$, we have $R \backslash Q=T$, and so $P=Q$. Therefore $P \in \operatorname{Min}(I)$.

Conversely, if $P \in \operatorname{Min}(I)$, then $T=R \backslash P$ is an m-system, by [7, Corollary 10.4]. Furthermore, $T \cap I=\emptyset$. Thus $T \in \Gamma$. Suppose that there exists $S \in \Gamma$ such that $T \subseteq S$. Hence $S$ is an $m$-system and $S \cap I=\emptyset$. By Zorn's Lemma, there exists an ideal $I \subseteq Q$ which is maximal with respect to being disjoint from $S$. From [7, Proposition 10.5], it follows that $Q$ is a prime ideal. Since $S \cap Q=\emptyset$ and $T \subseteq S$, we have $Q \cap T=\emptyset$. Hence $I \subseteq Q \subseteq P$, and consequently $Q=P$, because $P \in \operatorname{Min}(I)$. It follows from $P \cap S=\emptyset$ that $S \subseteq R \backslash P=T$, and so $T=S$. Therefore, $T$ is a maximal element of $\Gamma$.

It is well known that, if $I$ is an ideal of a commutative ring $R$, then $P \in \operatorname{Min}(I)$ if and only if for each $a \in P$, there exist $c \in R \backslash P$ and $n \in \mathbb{N}$ such that $(a c)^{n} \in I$. We need generalization of this conclusion for the noncommutative rings. In the following Lemma, we will generalize it to right duo rings.

Proposition 4.9. Let $R$ be a p-right duo ring and $I$ be a proper ideal of $R$. If $P$ is a nonzero prime ideal of $R$ containing $I$ and $T=R \backslash P$, then the following statements are equivalent:

(1) $P \in \operatorname{Min}(I)$.

(2) For every $x \in P$, there exist $y, z \in T$ and $n \in \mathbb{N}$ such that $y x^{n} z \in I$.

Proof. $1 \Rightarrow 2$. Let $P \in \operatorname{Min}(I)$ and $0 \neq x \in P$. If

$$
\Gamma=\{S \subseteq R \mid S \text { is an } m-\text { system and } S \cap I=\emptyset\} \text {, }
$$


then $T$ is a maximal element of $\Gamma$, by Proposition 4.8. Now, we assume that

$$
T^{\prime}=\left\{y x^{n} z \mid y, z \in T, n \in \mathbb{N} \cup\{0\}\right\} .
$$

Let $y_{1} x^{m} z_{1}, y_{2} x^{n} z_{2} \in T^{\prime}$. From Lemma 2.2, it is clear that $z_{1} y_{2} \in T$, and hence there is an element $r \in T$ such that $z_{1} y_{2} x^{n}=x^{n} r$, because $R$ is a $p$-right duo ring. Thus

$$
y_{1} x^{m} z_{1} y_{2} x^{n} z_{2}=y_{1} x^{m+n} r z_{2} \in T^{\prime} .
$$

Therefore $T^{\prime}$ is an $m$-system. Obviously, $x \in T^{\prime} \backslash T$, consequently $T \varsubsetneqq T^{\prime}$. Hence $T^{\prime} \notin \Gamma$, by the maximality of $T$. However, this yields $T^{\prime} \cap I \neq \emptyset$. Therefore, there exist $y, z \in T$ and $n \in \mathbb{N}$ such that $y x^{n} z \in I$.

$2 \Rightarrow 1$. Let $Q$ be a prime ideal of $R$ such that $I \subseteq Q \subseteq P$. For each $x \in P$, there exist $n \in \mathbb{N}$ and $y, z \in T$ such that $y x^{n} z \in I \subseteq Q$, by hypothesis. Since $Q$ is a prime ideal and $y, z \notin Q$, we have $x \in Q$, by Lemma 2.2. Therefore $Q=P$, and so $P \in \operatorname{Min}(I)$.

In the following Proposition, which is an analogue of [1, Lemma 2.1], we give conditions that, whenever $J$ is a left ideal, then every minimal prime ideal of a left $z_{J}$-ideal, is also a left $z_{J}$-ideal.

Proposition 4.10. Let $R$ be a p-right duo ring, $I$ be an ideal and $J$ be a left ideal of $R$. If $I$ is a left $z_{J}$-ideal, then every minimal prime ideal of $I$ is a left $z_{J}$-ideal.

Proof. Let $P \in \operatorname{Min}(I)$. For every element $a \in P$, there exist elements $b, c \in R \backslash P$ and $n \in \mathbb{N}$ such that $b a^{n} c \in I$, by Proposition 4.9. Since $I$ is a left $z_{J}$-ideal, we have $M_{b a^{n} c} \cap J \subseteq I$. Now, it follows from Lemma 2.5 that

$$
M_{b} \cap M_{a} \cap M_{c} \cap J=M_{b} \cap M_{a^{n}} \cap M_{c} \cap J=M_{b a^{n} c} \cap J \subseteq I \subseteq P .
$$

Obviously, $M_{b}, M_{c} \nsubseteq P$, because $b, c \notin P$. It follows that $M_{a} \cap J \subseteq P$, by Lemma 3.11, and so $P$ is a left $z_{J}$-ideal of $R$.

Proposition 4.11. Let $R$ be a right duo ring and $J$ be a left ideal of $R$. If $P$ is a prime ideal of $R$ for which $J \nsubseteq P$, then $P$ is a left $z_{J}$-ideal if and only if $P$ is a $z$-ideal.

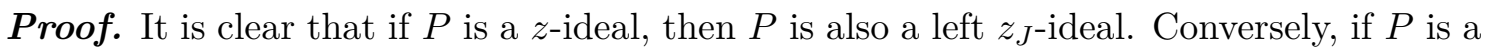
left $z_{J}$-ideal, then $M_{a} \cap J \subseteq P$, for each $a \in P$. Since $J \nsubseteq P$, Lemma 3.11 yields $M_{a} \subseteq P$, for each $a \in P$. Hence $P$ is a $z$-ideal of $R$.

Let $R$ be a $p$-right duo ring and $I$ be an ideal of $R$. From Lemma 2.2, it is clear that $\sqrt{I} \subseteq P$, for each $P \in \operatorname{Min}(I)$. On the other hand, if $P \in \operatorname{Min}(I)$ and $x \in R \backslash P$, we conclude from Proposition 4.9 that $x^{n} \notin I$, for every $n \in \mathbb{N}$, and so $x \notin \sqrt{I}$. Therefore, we have

$$
\sqrt{I}=\bigcap_{P \in \operatorname{Min}(I)} P .
$$

The following Lemma corresponds to [1, Lemma 2.2].

Lemma 4.12. Let $R$ be a p-right duo ring, $I$ be an ideal and $J$ be a left ideal of $R$. If $I$ is a left $z_{J}$-ideal, then $I_{z} \cap J \subseteq I$.

Proof. As we have seen in the preceding paragraph

$$
\sqrt{I}=\bigcap_{P \in \operatorname{Min}(I)} P .
$$

From Proposition 3.9, it follows that

$$
I_{z} \cap J=(\sqrt{I})_{z} \cap J=\left(\bigcap_{P \in \operatorname{Min}(I)} P\right)_{z} \cap J .
$$


Moreover, Lemma 3.6 yields

$$
\left(\bigcap_{P \in \operatorname{Min}(I)} P\right)_{z} \subseteq \bigcap_{P \in \operatorname{Min}(I)} P_{z}
$$

Thus

$$
I_{z} \cap J \subseteq\left(\bigcap_{P \in \operatorname{Min}(I)} P_{z}\right) \cap J .
$$

Since $I$ is a left $z_{J}$-ideal, from Proposition 4.10 , we see that $P$ is a left $z_{J}$-ideal, for every $P \in \operatorname{Min}(I)$. However, we conclude from Proposition 4.11 that $J \subseteq P$ or $P$ is a $z$-ideal, for every $P \in \operatorname{Min}(I)$.

We now assume that $P \in \operatorname{Min}(I)$. If $P$ is a $z$-ideal, then $P_{z}=P$, and so $P_{z} \cap J=P \cap J$. If $J \subseteq P$, then we also have $P_{z} \cap J=J=P \cap J$. Therefore

$$
\left(\bigcap_{P \in \operatorname{Min}(R)} P_{z}\right) \cap J=\left(\bigcap_{P \in \operatorname{Min}(R)} P\right) \cap J=\sqrt{I} \cap J
$$

and from (4.1) we get

$$
I_{z} \cap J \subseteq \sqrt{I} \cap J .
$$

Let us finally prove that $\sqrt{I} \cap J \subseteq I$. If $x \in \sqrt{I} \cap J$, then there is a positive integer $n$ such that $x^{n} \in I$. Since $I$ is a left $z_{J}$-ideal, we have $M_{x^{n}} \cap J \subseteq I$. Hence, from Lemma 2.5, it follows that

$$
x \in M_{x} \cap J=M_{x^{n}} \cap J \subseteq I .
$$

Thus $\sqrt{I} \cap J \subseteq I$, and consequently $I_{z} \cap J \subseteq \sqrt{I} \cap J \subseteq I$.

Lemma 4.13. Let $R$ be a p-right duo ring and $J$ be a left ideal of $R$. If $I$ is an ideal of $R$, then $I$ is a left $z_{J}$-ideal if and only if $I$ is a left $z_{I+J}$-ideal.

Proof. If $I$ is a left $z_{I+J}$-ideal, then clearly $I$ is a left $z_{J}$-ideal. Conversely, let $I$ be a left $z_{J}$-ideal. Since $I \subseteq I_{z}$, from Lemma 4.12 and modular law follow that

$$
I_{z} \cap(I+J)=I+\left(I_{z} \cap J\right) \subseteq I .
$$

For every $a \in I$, we have $M_{a} \subseteq I_{z}$, because $I \subseteq I_{z}$ and $I_{z}$ is a $z$-ideal. Hence

$$
M_{a} \cap(I+J) \subseteq I_{z} \cap(I+J) \subseteq I .
$$

Therefore, $I$ is a left $z_{I+J^{-}}$ideal.

Lemma 4.14. Let $R$ be a right duo ring. If $I$ and $J$ are two left ideals of $R$ such that at least one of them is ideal, then $I \cap J$ is a left $z_{J}$-ideal if and only if $I$ is a left $z_{J}$-ideal.

Proof. We first assume that $J$ is an ideal. If $I$ is a left $z_{J}$-ideal, then for every $a \in I \cap J$, we have $M_{a} \cap J \subseteq I$, and so $M_{a} \cap J \subseteq I \cap J$. Hence $I \cap J$ is a left $z_{J}$-ideal.

Conversely, let $I \cap J$ be a left $z_{J}$-ideal and $a \in I$. We must show that $M_{a} \cap J \subseteq I$. We now assume that $x \in M_{a} \cap J$. Thus $x a \in I \cap J$, because $J$ is an ideal. Since $I \cap J$ is a left $z_{J}$-ideal, $M_{x a} \cap J \subseteq I \cap J$. From Lemma 2.5 , we see that

$$
x \in M_{x} \cap M_{a} \cap J=M_{x a} \cap J \subseteq I \cap J \subseteq I .
$$

Therefore $M_{a} \cap J \subseteq I$, and so $I$ is a left $z_{J}$-ideal.

Now, if $I$ is an ideal, then we can prove this Lemma by a similar argument.

The following result is an analogue of [1, Proposition 2.5].

Proposition 4.15. Let $R$ be a p-right duo ring and $M$ be a maximal ideal of $R$. If $I$ is an ideal of $R$, then $I$ is a $z$-ideal if and only if $I \cap M$ is a $z$-ideal. 
Proof. If $I$ is a $z$-ideal, then clearly $I \cap M$ is a $z$-ideal. We now assume that $I \cap M$ is a $z$-ideal of $R$. If $I \subseteq M$, then $I=I \cap M$, and so $I$ is a $z$-ideal. If $I \nsubseteq M$, then $M_{a} \subseteq I \cap M$, for every $a \in I \cap M$, and so $M_{a} \cap M \subseteq I \cap M$. Thus $I \cap M$ is a left $z_{M}$-ideal. It follows from Lemma 4.14 that $I$ is a left $z_{M}$-ideal. Now, Lemma 4.13 implies that $I$ is a left $z_{R}$-ideal, because $I+M=R$. However, $I$ is a $z$-ideal of $R$.

The following Proposition is an analogue of [1, Proposition 2.6] and [2, Proposition 2.2]. Proposition 4.16. Let $R$ be a p-right duo ring and $J$ be an ideal of $R$ with $J \nsubseteq \operatorname{rad}(R)$. If $J$ is not a z-ideal, then there exists an ideal $I$ of $R$ such that $I \varsubsetneqq J$ and $I$ is a left $z_{J}$-ideal which is not a $z$-ideal.

Proof. Since $J \nsubseteq \operatorname{rad}(R)$, there is a maximal ideal $M$ of $R$ such that $J \nsubseteq M$. Thus $I=J \cap M$ is an ideal of $R$ and $I \varsubsetneqq J$. Obviously, for every $a \in I, M_{a} \cap J \subseteq M \cap J=I$, and so $I$ is a left $z_{J}$-ideal of $R$. From Proposition 4.15, it follows that $J$ is a $z$-ideal if and only if $I$ is a $z$-ideal. Therefore, the desired conclusion trivially holds.

Lemma 4.17. Let $R$ be a p-right duo ring and $I$ be an ideal of $R$. If $I$ is a left relative $z$-ideal of $R$, then the set

$$
\Gamma=\{J \mid J \text { is a z-factor of } I\}
$$

has a maximal member with respect to inclusion. Furthermore, every maximal element of $\Gamma$ properly contains $I$.

Proof. Obviously, $\Gamma \neq \emptyset$. If $\Sigma$ is a non-empty totally ordered subset of $\Gamma$, then clearly $L=\bigcup_{J \in \Sigma} J$ is a left ideal which $L \nsubseteq I$. We will show that $I$ is a $z_{L}$-ideal. For every $a \in I$, we have

$$
M_{a} \cap L=M_{a} \cap\left(\bigcup_{J \in \Sigma} J\right)=\bigcup_{J \in \Sigma}\left(M_{a} \cap J\right) \subseteq I,
$$

because $J$ is a $z$-factor of $I$, for all $J \in \Sigma$, and so $M_{a} \cap L \subseteq I$. Hence $I$ is a left relative $z_{L}$-ideal, and consequently $I$ is an upper bound for $\Sigma$ in $\Gamma$. From Zorn's Lemma, we see that $\Gamma$ has a maximal element.

Now, we show that every maximal element of $\Gamma$ properly contains $I$. If $J$ is a maximal element of $\Gamma$, then $I$ is a left $z_{J}$-ideal and $J \nsubseteq I$. Hence $I$ is a left $z_{I+J}$-ideal, by Lemma 4.13. Since $I+J \nsubseteq I, I+J$ is a $z$-factor of $I$, and so $I+J \in \Gamma$. Therefore, by the maximality of $J$, we deduce that $J=I+J$, and consequently $I \varsubsetneqq J$.

Lemma 4.18. Let $R$ be a right duo ring and $I, J$ and $L$ be left ideals of $R$ such that

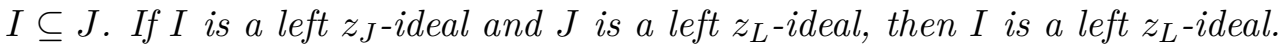

Proof. Since $I$ is a left $z_{J}$-ideal, we have $M_{a} \cap J \subseteq I$, for every $a \in I$. Moreover, $M_{a} \cap L \subseteq$ $J$, for every $a \in I$, because $I \subseteq J$ and $J$ is a left $z_{L}$-ideal. Thus $M_{a} \cap L \subseteq M_{a} \cap J \subseteq I$, for every $a \in I$. Therefore $I$ is a left $z_{L}$-ideal.

Theorem 4.19. Let $R$ be a duo ring such that every proper ideal of $R$ is a left relative $z$-ideal. If $R$ is a p-right duo ring, then every ideal of $R$ is a z-ideal.

Proof. It is clear that $R$ is a $z$-ideal. Let $I$ be a proper ideal of $R$. Then $I$ is a left relative $z$-ideal, by hypothesis. It follows from Lemma 4.17 that there exists a maximal $z$-factor $J$ of $I$ such that $I \varsubsetneqq J$. We claim that $J=R$. If $J \neq R$, then $J$ is also a left relative $z$-ideal, and hence we can assume that $L$ is a $z$-factor of $J$ such that $J \varsubsetneqq L$, by Lemma 4.17. It follows that $I$ is a left $z_{J}$-ideal and $J$ is a left $z_{L}$-ideal. From Lemma 4.18 , we may conclude that $I$ is a left $z_{L}$-ideal. Since $I \varsubsetneqq J \varsubsetneqq L, L$ is a $z$-factor of $I$, which contradicts the maximality of $J$. Therefore, $J=R$, and so $I$ is a $z$-ideal.

Acknowledgment. We would like to thank the referee for the valuable suggestions and comments. 


\section{References}

[1] A.R. Aliabad, F. Azarpanah and A. Taherifar, Relative z-ideals in commutative rings, Comm. Algebra, 41, 325-341, 2013.

[2] F. Azarpanah and A. Taherifar, Relative z-ideals in $C(X)$, Topology Appl. 156, 1711-1717, 2009

[3] R.C. Courter, Finite dimensional right duo algebras are duo, Proceedings of the Amer. Math. Soc. 84 (2), 157-161, 1982.

[4] L. Gillman and M. Jerison, Rings of continuous functions, The University Series in Higher Mathematics, New York, Van Nostrand, 1960.

[5] N.K. Kim and Y. Lee, On a ring property unifying reversible and right duo rings, J. Korean Math. Soc. 50 (5), 1083-1103, 2013.

[6] C.W. Kohls, Ideals in rings of continuous functions, Fund. Math. 45, 28-50, 1957.

[7] T.Y. Lam, A first course in noncommutative ring, Graduate Texts in Mathematics 131, Springer-Verlag, New York, 1991.

[8] T.Y. Lam and A.S. Dugas, Quasi-duo rings and stable range descent, J. Pure Appl. Algebra 195, 243-259, 2005.

[9] G. Marks, Duo rings and ore extensions, J. Algebra, 280, 463-471, 2004.

[10] G. Mason, z-ideals and prime ideals, J. Algebra, 26, 280-297, 1973.

[11] G. Mason, Prime z-ideals of $C(X)$ and related rings, Canad. Math. Bull. 23 (4), 437-443, 1980.

[12] A. Rezaei Aliabad and R. Mohamadian, On z-ideals and $z^{\circ}$-ideals of Power Series Rings, J. Math. Ext. 7 (2), 93-108, 2013. 